\title{
Prediction of Single Neuron Spiking Activity using an Optimized Nonlinear Dynamic Model
}

\author{
Anish Mitra*, Andre Manitius** and Tim Sauer***
}

\begin{abstract}
The increasing need of knowledge in the treatment of brain diseases has driven a huge interest in understanding the phenomenon of neural spiking. Researchers have successfully been able to create mathematical models which, with specific parameters, are able to reproduce the experimental neuronal responses. The spiking activity is characterized using spike trains and it is essential to develop methods for parameter estimation that rely solely on the spike times or interspike intervals (ISI). In this paper we describe a new technique for optimization of a single neuron model using an experimental spike train from a biological neuron. We are able to fit model parameters using the gradient descent method. The optimized model is then used to predict the activity of the biological neuron and the performance is quantified using a spike distance measure.
\end{abstract}

\section{INTRODUCTION}

Neurons form the basic building block of our brain and central nervous system. Networks of neurons communicate with each other to perform complicated tasks. This communication amongst neurons is through the 'spiking' of the membrane potential [1]. Over the last 60 years there has been extensive research to model the spiking phenomenon. One of the popular classes of models used are nonlinear dynamical systems. In 1952, Hodgkin and Huxley developed a model [2] which was able to reproduce the membrane potential of a giant squid axon. The model consists of four differential equations and a number of parameters that relate to physiological variables such as gating currents, ion concentrations and conductances. This makes the model complex and computationally expensive. Since then there has been a large number of efforts to develop models which can reproduce the full range of spiking behavior but are simple in nature and computationally inexpensive. [3] summarizes few successful models and compares them based on previously mentioned attributes. The Izhikevich model [4] and the augmented multi-timescale adaptive threshold (AugMAT) model ([5], [6]) are two such models which outperform the rest. Another class of models present in literature are those based on stochastic processes such as renewal theory or Markov chain processes [7].

Following the success of these different single neuron models, the problem of parameter fitting has drawn huge

\footnotetext{
*A. Mitra is with the Department of Electrical and Computer Engineering , George Mason University, Fairfax, VA 22030 USA amitralegmu.edu

**A. Manitius is with the Faculty of Department of Electrical and Computer Engineering, George Mason University, Fairfax, VA 22030 USA amanitiuegmu.edu

***T. Sauer is with the Faculty of Department of Mathematical Sciences, George Mason University, Fairfax, VA 22030 USA t saueregmu. edu
}

attention. Efforts are being made to accurately estimate model parameters from experimental data using innovative methods. The unscented kalman filter (UKF) [8] is one of the successful methods for estimating the state of a nonlinear system based on observed measurements. A summary of the efforts to use kalman filters to estimate the spiking of a neuron can be found in [9]. However, such methods require the nonlinear models to satisfy the observability condition. A different approach to estimate parameters or states is to develop a cost function and use optimization techniques to find the solution which gives the minimum cost. [10] summarizes the efforts in this area and mentions the different methods currently being used. It is interesting to note that in a few cases even a simple 'brute force' technique is found to be effective. This suggests that there is still a huge scope of research in this area, mainly to develop automated techniques which can fit models to neural spike trains.

In this paper, we describe a novel approach to neuron model optimization by introducing a new technique to compare spike trains of single neurons. We are able to estimate the parameters of the model by formulating a nonlinear optimization problem using the proposed performance function. The optimal solution is found by using the gradient descent method. The optimized model is then simulated to predict the spiking activity of the neuron. The spike trains (of the model and biological neuron) are compared using the bivariate SPIKE-distance function [11].

\section{THE PROBLEM}

The purpose of this paper is to be able to successfully predict the spiking activity of a biological neuron. There are numerous mathematical models which can reproduce the neuronal response. The problem is, therefore, to tune the parameters of the model based on observed data. The three major aspects of this problem are (i) the model, (ii) the comparison technique and (iii) the method of optimization.

\section{A. Nonlinear Model :}

We have used the augmented version of the Multitimescale Adaptive Threshold (AugMAT) model [6]. This model is able to reproduce the different spiking behaviors of a biological neuron, and is computationally inexpensive at the same time. It is described by two differential equations. The first equation represents the membrane potential, $v(t)$ and the other is the adaptive threshold, $\theta(t)$. The neuron spikes if the membrane potential becomes greater than the threshold. Immediately after spiking the threshold is reset.

In Eq. $1, t_{i}$ is the $i$ th spike time, $\mathrm{L}$ is the number of 
threshold time constants, $\tau_{j}(j=1 . . L)$ is the $j$ th time constant, $\alpha_{j}$ is the weight corresponding to $\tau_{j}$ and $\omega$ is the resting value. The time constants $\tau_{j}, \tau_{m}, \tau_{v}$ and $R$ are fixed.

$$
\begin{aligned}
\tau_{m} \frac{d V}{d t} & =-V+R I(t) \\
\theta(t) & =\sum_{i} H\left(t-t_{i}\right)+\beta \int_{0}^{t} K(s) V^{\prime}(t-s) d s+\omega \\
H(t) & =\sum_{j=1}^{L} \alpha_{j} e^{-t / \tau_{j}} \\
K(s) & =s e^{-s / \tau_{v}}
\end{aligned}
$$

In this paper we have chosen $L$ and the 'fixed' parameters as suggested by [6].

$$
\begin{aligned}
& \tau_{m}=10 \mathrm{~ms}, R=50 \mathrm{M} \Omega \\
& \tau_{v}=5 \mathrm{~ms}, L=2 \\
& \tau_{1}=10 \mathrm{~ms}, \tau_{2}=200 \mathrm{~ms}
\end{aligned}
$$

The input to the model, $I(t)$ should be in $n A$. In the process of model optimization, $\alpha_{1}, \alpha_{2}, \omega$ and $\beta$ are estimated. Initial condition, $v\left(t_{0}\right)=0$ is fixed and $\theta\left(t_{0}\right)=\theta_{0}$ is estimated. In total, there are five variable parameters for the model considered in this paper. Fig. 1 shows a simulation of the model, and the computation of the spike train from the states of the differential equations. The set of spike times, $t_{k}$, is defined as

$$
\begin{aligned}
\epsilon(t) & =V(t)-\theta(t) \\
t_{k}: \epsilon\left(t_{k}\right) & =0 \wedge \epsilon^{\prime}\left(t_{k}\right)>0
\end{aligned}
$$
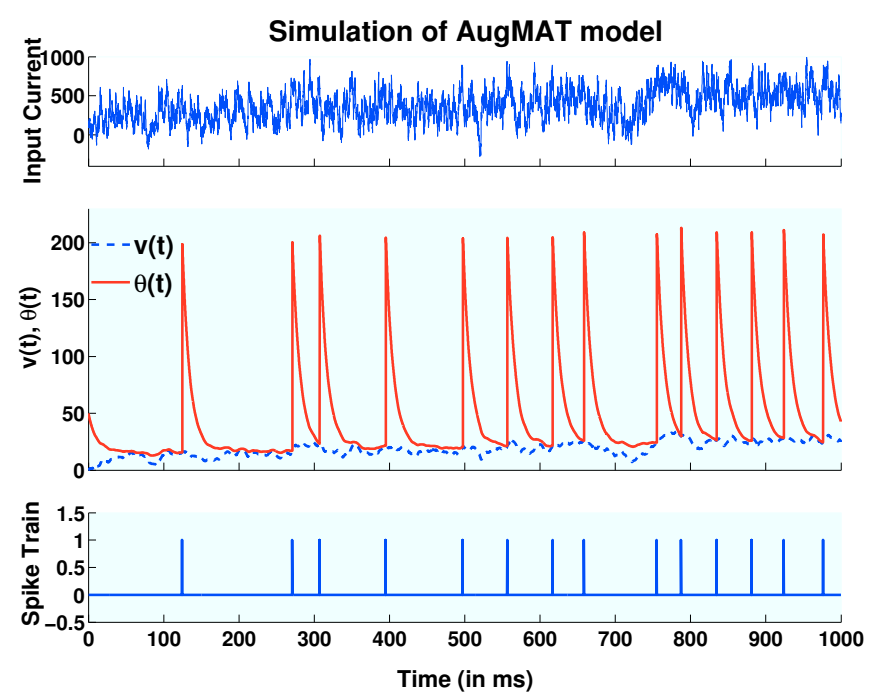

Fig. 1. $\left[\alpha_{1}=180, \alpha_{2}=3, \beta=0.2, \omega=15\right]$ The top row is the input current. It is a sum of different exponentials, to reproduce the type of stimulus a biological neuron receives from its dendritic tree. The second row shows $v(t)$ (blue, dashed) and $\theta(t)$ (red, solid) functions. The last row shows the constructed spike train, which is a binary string of $1 \mathrm{~s}$ and $0 \mathrm{~s}$

\section{B. Performance function :}

We propose a new performance function that takes into consideration the number of spikes in each spike train as well as the synchronization of these spikes. The surface was computed as a function of changing parameters and was found to be smooth. It is not a complex function, and hence does not affect the speed of optimization.

The spike times are extracted from the spike train,

$$
t_{\text {spikes }}=\left[\begin{array}{lllll}
t_{1} & t_{2} & \cdot & \cdot & t_{n}
\end{array}\right]
$$

where $t_{1}, t_{2}, .$. are the time of spikes and $n$ is the total number of spikes in the spike train of length $t_{f}$. A 'staircase' is given by

$$
\psi(t)=\sum_{k=1}^{n} v\left(t-t_{k}\right)
$$

where $v(x)$ is the step function which is equal to 1 for $x>0$ and 0 otherwise.

Fig. 2 gives a graphical illustration of how $\psi(t)$ is created from the spike train. The error, $\xi$, is given by the square of the difference between the area of the staircase function, $\psi(t)$, of each model.

$$
\xi=\frac{1}{t_{f}^{2}} \int_{0}^{t_{f}}\left[\psi_{1}(t)-\psi_{2}(t)\right]^{2} d t
$$

$\psi_{1}(t)$ and $\psi_{2}(t)$ are the staircase functions of two different spike trains.

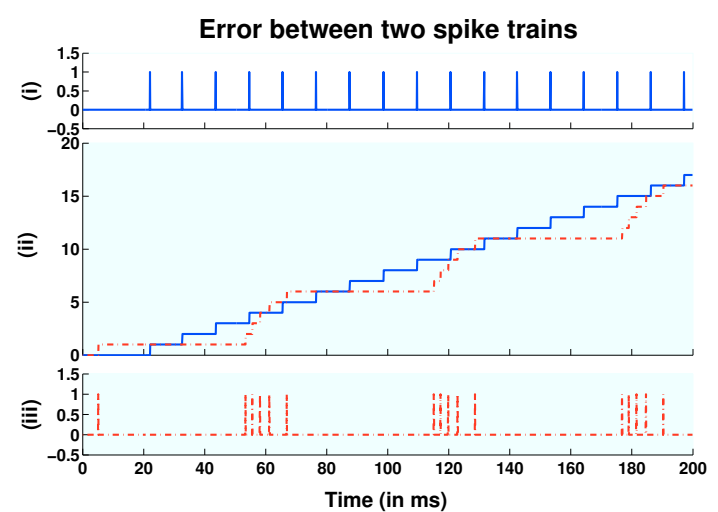

Fig. 2. Comparison of two neuron models using the performance function defined in Eq. 4. (i) Spike train of model 1 (Regular Spiking) (ii) $\psi_{1}(t)$ (blue solid line), $\psi_{2}(t)$ (red dotted line). The error, $\xi$, is the square of the area between the two lines (iii) Spike train of model 2 (Bursting)

The proposed error function is used to fit model parameters to the experimental data (explained in section III). The model is then simulated with the given input current. To evaluate the performance of the model and synchrony of the synthetic and experimental spike train, we use the bivariate SPIKE-distance described by Kreuz et al. in [11]. This comparison technique computes the instantaneous differences between the ISIs. The spike distance is defined as the temporal average of the time profile. The value is normalized between 0 and 1 , with 0 respresenting two exactly same spike trains.

\section{MODEL OPTIMIZATION}

There are numerous iterative algorithms available in the literature of numerical optimization [12] to search the minimum of a function $(\xi)$. Gradient based algorithms compute the gradient of the function with respect to the parameter vector, $\mathbf{p}$. The vector is then updated using the gradient. If 
the update, $\hat{\mathbf{p}}$ is in the opposite direction of the gradient, then by updating the vector iteratively, it converges to a minima. To reach a maxima, the update should be in the direction of the gradient.

$$
\hat{\mathbf{p}}=\mathbf{p}-\nu \frac{\partial \xi}{\partial p}
$$

$\nu$ is the step size of the gradient based search. For the problem defined in the previous section, the performance function, $\xi$, is given by Eq. 4 and the parameter vector consists of the variable parameters of the AugMAT model.

$$
\mathbf{p}=\left[\alpha_{1}, \alpha_{2}, \beta, \omega, \theta_{0}\right]^{T}
$$

Computation of the gradient requires analytical formulae of the derivatives of the performance function with respect to each parameter.

$$
\begin{gathered}
\frac{\partial \xi}{\partial p}=\frac{1}{t_{f}^{2}} \int_{0}^{t_{f}} 2\left[\psi(t)-\psi^{d}(t)\right] \frac{\partial \psi(t)}{\partial p} d t \\
\frac{\partial \psi(t)}{\partial p}=\sum_{k=1}^{M} \delta\left(t-t_{k}\right)\left[-\frac{\partial t_{k}}{\partial p}\right]
\end{gathered}
$$

In Eq. 7, the $\frac{\partial t_{k}}{\partial p}$ expression represents the change in the $k^{t h}$ spike time with respect to the change in parameter $p$. A similar computation has been done by Booij and Nguyen in [13]. Applying the total differential identity to the variable $\epsilon(t)$ (Eq. 2) in our problem,

$$
\begin{gathered}
\epsilon^{\prime}\left(t_{k}\right) d t_{k}+\frac{\partial \epsilon\left(t_{k}\right)}{\partial p} d p=0 \\
\frac{\partial t_{k}}{\partial p}=\frac{-\frac{\partial \epsilon\left(t_{k}\right)}{\partial p}}{\epsilon^{\prime}\left(t_{k}\right)} \\
\epsilon^{\prime}\left(t_{k}\right)=\frac{\epsilon\left(t_{k}+\delta\right)-\epsilon\left(t_{k}\right)}{\delta} \\
\frac{d \epsilon\left(t_{k}\right)}{d p}=\frac{d V\left(t_{k}\right)}{d p}-\frac{d \theta\left(t_{k}\right)}{d p} \\
\frac{d V\left(t_{k}\right)}{d p}=0 \\
\frac{d \theta\left(t_{k}\right)}{d p}=\frac{\partial \theta\left(t_{k}\right)}{\partial p}+\sum_{i: t_{i}<t_{k}} \frac{\partial \theta\left(t_{k}\right)}{\partial t_{i}} \frac{\partial t_{i}}{\partial p}
\end{gathered}
$$

Combining Eqns. 5-10, the gradient of the proposed performance function can be computed for each element in the parameter vector $\mathbf{p}$.

\section{RESULTS}

Experimental data (of an L5 neuron) was obtained from the International Neuroinformatics Coordinating Facility (INCF) [14] ${ }^{1}$. A single neuron was stimulated and the membrane potential was recorded. There were a total of 13 trials performed and the current and voltage traces were

\footnotetext{
${ }^{1}$ http://www.incf.org/community/competitions/spike-timeprediction/2009/challenge-a
}

recorded and made publicly available. We used this data to optimize the AugMAT model, and evaluate the predictive performance.

The model was optimized using a $4 \mathrm{~s}$ spike train where the neuron was stimulated with constant current. The spike times were extracted and used to estimate parameters of the model. The parameter space was chosen by setting minimum and maximum values, taking into consideration the nature of spiking. Different parameter sets from this space were able to generate the different kinds of responses.

$$
\begin{aligned}
\chi= & {\left[\left\{\alpha_{1}, \alpha_{2}, \beta, \omega, \theta_{0}\right\}: 100<\alpha_{1}<220,0<\alpha_{2}<8,\right.} \\
& \left.0.1<\beta<0.5,5<\omega<15,0<\theta_{0}<100\right]
\end{aligned}
$$

100 random samples were chosen from the parameter space. Each sample serves as an initial parameter vector for the optimization algorithm. The optimal parameter vector for each starting point was found using the gradient descent method (GD). The number of iterations was set at 20 , since it was observed that there was no substantial improvement in the error after 20 iterations. These estimated parameters were then used to simulate the model. The simulated spike train was compared to the experimental spike train using the bi-variate SPIKE-distance [11].

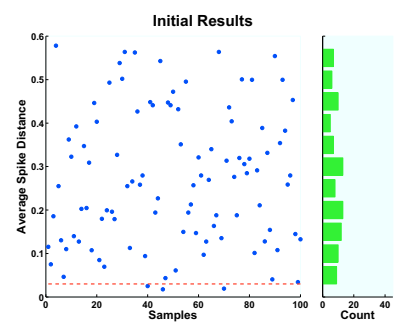

(a) Mean SPIKE-distance $=0.28$

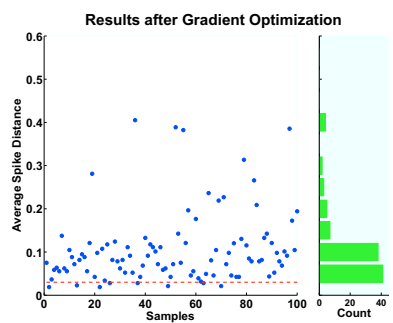

(b) Mean SPIKE-distance $=0.10$
Fig. 3. Prediction results : (a) shows the initial distribuition of the SPIKE-distance measure (100 samples) and (b) shows the distribution after optimization is achieved. The red dotted line shows the multi-variate distance measure over the 13 different trials of the same experimental neuron

Fig. 3 shows the initial and final bi-variate spike distances computed for each sample. The distribution of the spike distances for the initial population was $\mathbf{0 . 2 8} \pm 0.16$. After optimization, the final spike distances were $\mathbf{0 . 1 0} \pm 0.08$. $62 \%$ of the final predicted spike trains had a SPIKE-distance of less than 0.1. As a benchmark, the multi-variate SPIKEdistance for the 13 trials of the L5 neuron was $\mathbf{0 . 0 3}$, which is indicative of the variability in the experimental neuron. This indicates that the results achieved are meaningful keeping in consideration the inaccuracies of the mathematical model itself.

As a comparison to our optimization technique, we also used the Nelder-Mead (NM) [15] method. The same initial population (as in GD) was taken and the model parameters were estimated by minimizing the SPIKE-distance using NM. We found that the prediction results were $0.13 \pm 0.12$. The GD method also took less time as compared to the NM method. Fig. 4 shows the spiking activity of the biological neuron and the optimized model. The results of the SPIKEdistance between the predicted and actual spike trains for the 


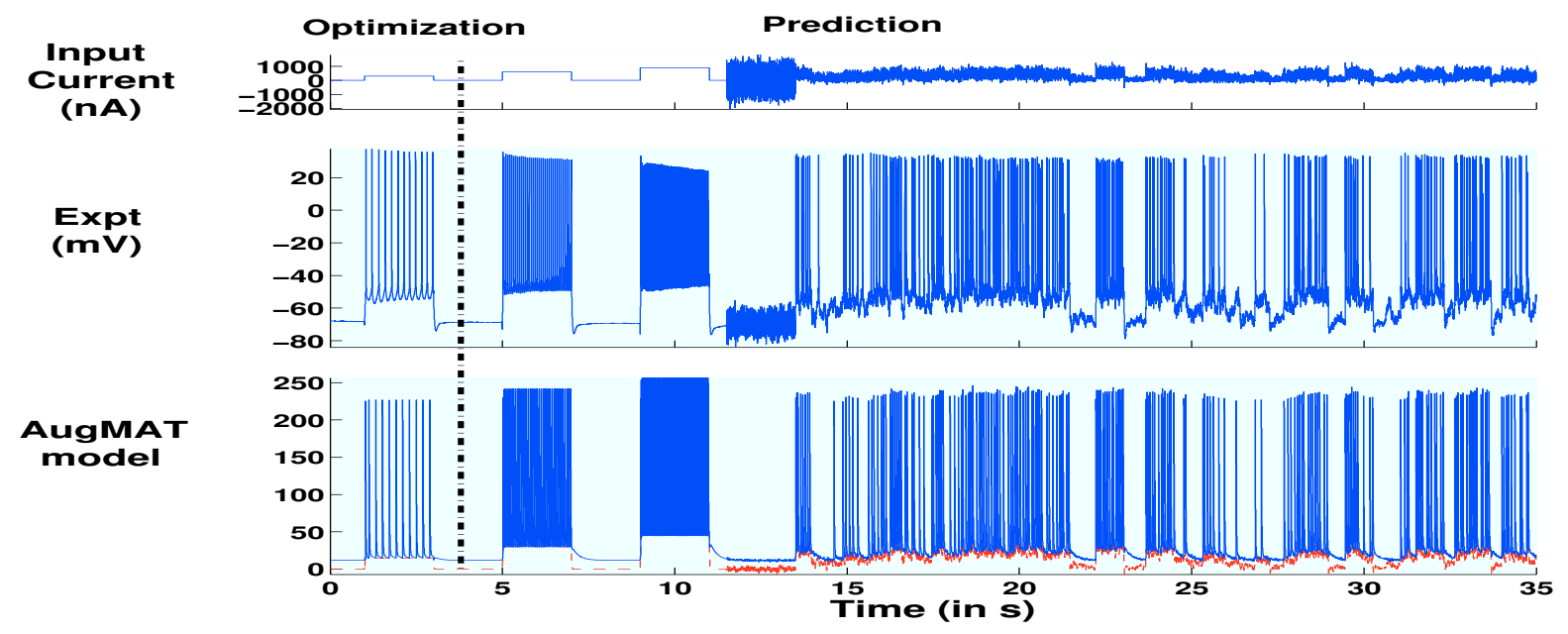

Fig. 4. The first $4 \mathrm{~s}$ (left of the vertical partition line) is used for model optimization. The model (bottom row) $\left[\alpha_{1}=183.4, \alpha_{2}=2.53, \beta=0.087, \omega=\right.$ $\left.11.93, \theta_{0}=58.2\right]$ is then simulated using the input current provided (top row) and compared to the experimental data (middle row)

100 samples are summarized in Table 1.

TABLE I

PREDICTION RESULTS (SPIKE-DisTANCE) FOR 100 SAMPLES

\begin{tabular}{cccc} 
& Initial & After GD & After NM \\
\hline Mean & 0.28 & 0.10 & 0.13 \\
Std. Dev. & 0.16 & 0.08 & 0.12 \\
\hline
\end{tabular}

\section{CONCLUSIONS AND FUTURE WORK}

\section{A. Summary}

Our objective was to predict the neural spiking activity of a biological neuron using mathematical models. We optimized the model by computing the error between the model and the experimental spike trains using the proposed 'staircase' function. Using the gradient descent technique, we were able to find optimal parameters which minimized this error. We then simulated the AugMAT model with the estimated parameters, and compared the predicted spike train to that of the experimental neuron using the SPIKE-distance function. Results showed that the final responses (spikes) were very 'close' to the biological neuron and we can conclude that the optimized model could be used as an efficent predictor of the neuron. Our method of optimization was also more accurate and faster than the Nelder-Mead method.

\section{B. Future work}

While our method successfully optimizes single neuron models, the objective is to characterize neuron populations and build predictors for a network of neurons interacting among themselves. Once the model of the network of neurons is estimated, it can be used to detect 'faults' in the experimental system. The algorithm can then be used in a closed loop form to predict and control brain activity. Potential applications are predicting and suppressing epileptic seizures before they occur, and controlling abnormal spiking patterns in the brain.

\section{REFERENCES}

[1] P. Dayan and L.F. Abbot, Theoretical neuroscience : computational and mathematical modeling of neural systems, The MIT Press; 2001.

[2] A. L. Hodgkin and A. F. Huxley, "The dual effect of membrane potential on sodium conductance in the giant squid axon of Loligo," The Journal of Physiology, vol. 116, no. 4, pp. 497-506, 1952.

[3] E. M. Izhikevich, "Which model to use for cortical spiking neurons?," IEEE Transactions on Neural Networks, vol. 15, no. 5, pp. 1063-1070, 2004.

[4] E. M. Izhikevich, "Simple model of spiking neurons," IEEE Transactions on Neural Networks, vol. 14, no. 6, pp. 1569-1572, 2003.

[5] R. Kobayashi, Y. Tsubo, S. Shinomoto, "Made-to-order spiking neuron model equipped with a multi-timescale adaptive threshold," Frontiers in Computational Neuroscience, vol. 3, no. 9, pp. 1-11, 2009.

[6] S. Yamauchi, H. Kim, S. Shinomoto, "Elemental spiking neuron model for reproducing diverse firing patterns and predicting precise firing times," Frontiers in Computational Neuroscience, vol. 5, no. 42, pp. $1-15,2011$.

[7] J. Tapson, C. Jin, A. van Schalke and R. Etienne-Cummings, "A First-Order Nonhomeogeneous Markov Model for the Response of Spiking Neurons Stimulated by Small Phase-Continuous Signals," Neural Computation, vol. 21, no. 6, pp. 1554-1588, 2009.

[8] E. A. Wan and R. Van der Merwe, "The unscented Kalman filter for nonlinear estimation," Adaptive Systems for Signal Processing, Communications, and Control Symposium, Lake Louise, Alta. , Canada, pp. $153-158,2000$.

[9] S. J. Schiff, "Kalman Meets Neuron: The Emerging Intersection of Control Theory with Neuroscience," 31st Annual International Conference of the IEEE EMBS, Minneapolis, Minnesota, USA, 2009.

[10] W. Van Geit, E. De Schutter and P. Achard, "Automated neuron model optimization techniques: a review," Biol Cybern, vol. 99, no. 4-5, pp. 241-251, 2008.

[11] T. Kreuz, D. Chicharro, M. Greschner and R. G. Andrzejak, "Timeresolved and time-scale adaptive measures of spike train synchrony," Journal of Neuroscience Methods, vol. 195, issue 1, pp. 92-106, 2011.

[12] I. Griva, S. G. Nash and A. Sofer, Linear and nonlinear optimization, Society for Industrial and Applied Mathematics; 2009.

[13] O. Booij and T. H. Nguyen, "A Gradient Descent Rule for Spiking Neurons Emitting Multiple Spikes," Information Processing Letters, vol. 95, no. 6, pp. 552-558, 2005.

[14] W. Gerstner and R. Naud, "How good are neuron models?," Science, vol. 326, no. 5951, pp. 379-380, 2009.

[15] J. A. Nelder and R. Mead, "A simplex method for function minimization," Computer Journal, vol. 7, no. 4, pp. 308-313, 1965. 